\title{
Quantum Entanglement: Can We "See" the Implicate Order? Philosophical Speculations
}

\author{
Michele Caponigro, Xiaojiang Jiang, Ravi Prakash \\ Ram Lakhan Pandey Vimal
}

\begin{abstract}
This brief paper argue about a possible philosophical description of the implicate order starting from a simple theoretical experiment. Utilizing an EPR source and the human eyes (as biological detectors) of a "single" person, we try to investigate the philosophical and physical implications of quantum entanglement in terms of implicate order. We know, that most specialists still disagree on the exact number of photons required to trigger a neural response, although there will be many technical challenges, we assume that neural response will be achieved in some way. The objective of paper is to investigate possible links between: quantum mechanics, quantum cognitive science, brain and mind. At the moment, the questions are more than the answers. We argue that we are perennially immersed in the implicate order and that the "real path" of quantum entanglement process is from the implicate order towards explicate order, not vice versa. Finally, we speculate about the common ground between the implicate order and chitta (Vedic theory of Mind).
\end{abstract}

Key Words: quantum entanglement, EPR source, human eyes entanglement, implicate explicate order, chitta

NeuroQuantology 2010; 3: 378-389

\section{Introduction}

In all of classical physics, the description of a system's state depends only on itself and its immediate surroundings, in other words, classical physics is local. This is not the underlying reality described by quantum mechanics. Quantum mechanics exhibits

Corresponding author: Michele Caponigro

Address: Piazzale S. Agostino 2, 24129 Bergamo, Italy. Bergamo University, Epistemology of Complexity, Department of Neurology, Research Institute of Surgery and Daping Hospital T.M. Medical University, 10 Changjiang Zhilu, Chongqing 400042, China. drravi2121@gmail.com. Central Institute of Psychiatry, India rlpvimal@yahoo.co.in. Vision Research Institute, 428 Great Road, Suite 11, Acton, MA 01720 USA

Phone: +390352052964

e-mail: michele.caponigro@unibg.it

Received: May 14, 2010. Revised: June 24, 2010.

Accepted: June 28, 2010. non-locality when it remains contextual even in this spatially separated setting. The phenomenon of quantum Entanglement is among the most counterintuitive concepts in quantum mechanics. Today the study of entanglement occupies a prime position in the field of quantum information processing. This term was first introduced by Schrödinger as the essence of quantum mechanics, the term describes a system composed of two or more particles, which exhibits the property that the results of measurements on one particle cannot be specified independently of the parameters of the measurements on the other particles. Although the different measurements can take place in spacelike separated regions, the 
results of each measurement depend on the complete experimental context of the whole system. From physical point of view, the entanglement is considered to be an objective property of the quantum system, but some experimental tests can demonstrate that the entanglement depends on the measurement context and therefore this entanglement becomes an entangled property itself. From philosophical point of view, there are possible "subjective" elements on the choice of the quantum system and their subsystems. In a recent paper (Torre, 2010) it is showed that a state that is factorizable in the Hilbert space corresponding to some choice of degrees of freedom, becomes entangled for a different choice of degrees of freedom. Therefore, entanglement is not a special case but is ubiquitous in quantum systems. According this work, as a consequence of this they conclude that in every state, even for those factorizable, we can find pairs of observables that will violate Bell's inequalities. The philosophical implications are that the appearance of entanglement depends on the choice of degrees of freedom can find an interesting application in the "disentanglement" of a state. Moreover, because of the fragility of entanglement, any interaction with the environment, which distinguishes between the entangled subsystems, collapses the quantum state. Therefore the decoherence (via interaction with the system's environment) play a central role in understanding the emergence of our classical world from quantum mechanics. Today an entanglement can be created, manipulated and quantified, bipartite entanglement is well understood and, it is also, accessible from an experimental viewpoint. Very interesting for the philosophical implications is the Entanglement swapping, a method that enables one to entangle two quantum systems that do not have direct interaction with one another. To conclude this brief survey, we can say that surely with the violation of Bell's inequality is started a tension between quantum mechanics and space-time theory. According Shimony (Shimony, 2007) there are other sources of tension, like the difficulty of quantizing general relativity and the difficulty of maintaining the very concept of a space-time continuum at the Planck level. He adds that a solution to the nonlocality problem created by Bell must be a deep solution. He believes that nonlocality is here to stay, but so far we only have a phenomenological account of it. What is needed is a deep theory underlying the phenomenology, in the way that Boltzmann's statistical account of thermodynamic processes provided the conceptual underpinning of the second law of thermodynamics.

\section{Our framework}

In this paper we are interested to address the question: can the human eyes (left and right eye) detect quantum entanglement? According Sekatski (Sekatski et al., 2009) "one" person could not detect entanglement by simply observing photons, for this reason they discuss about the possibility to test the quantum entanglement for several observers in order to demonstrate entanglement in a Bell-type experiment. The authors conclude that close to perfect threshold (human) detectors can be used to test quantum nonlocality without the need of any supplementary assumption. Our framework is different, we argue from theoretical point of view (without any specific technical discussion) about the possibility to test the quantum entanglement in a single person (his left and right eye as detectors). The picture (Fig.1) show how we can perform this test.

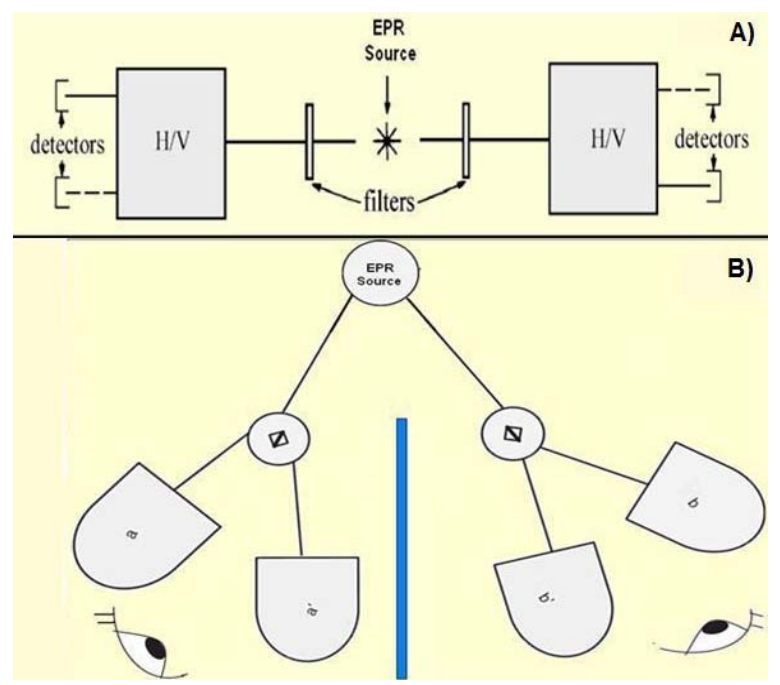

Figure 1. A. Classic EPR source application. B. Details of EPR source (entangled photons) and human eyes of single observer. 


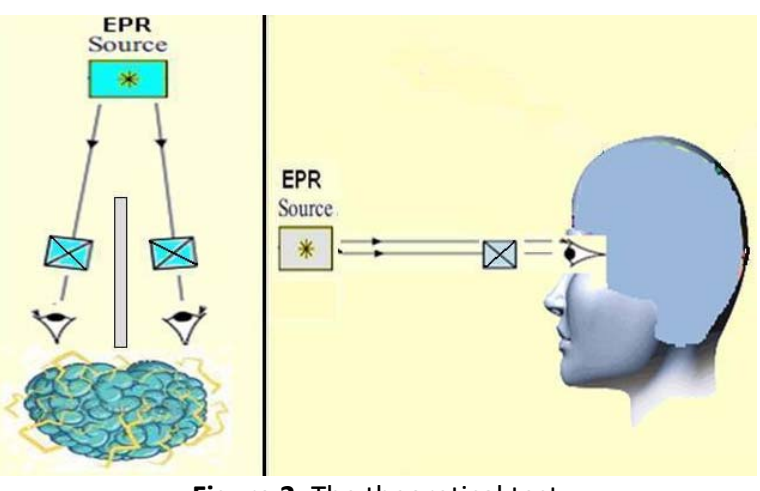

Figure 2. The theoretical test.

The "two" observers in this case are: Alice (left eye) and Bob (right eye). They receive entangled particles emitted by some source, each eye choose a measurement setting, a and b respectively, and then record their measurement outcomes values, say A and $\mathrm{B}$. The measurement process in this special case take place in the brain (Fig.2), but the real and interesting question is: "where the entanglement take place?" We will speculate philosophically on the (possible) quantum entanglement in terms of mind/brain and not in terms of quantum model of dissipative brain (Vitiello, 1995). We know that, at the moment the questions are more than the answers, for example:

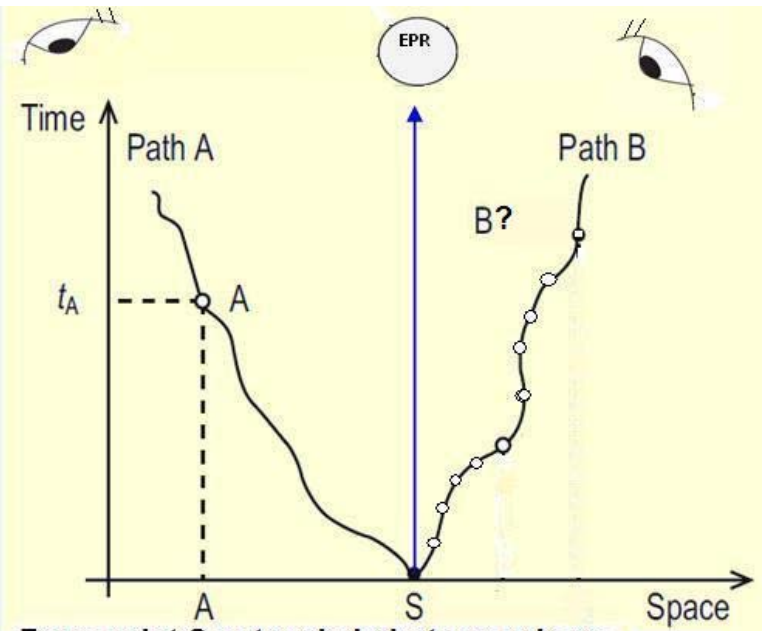

From point $S$ entangled photons pair are emitted in opposite directions.

A measurement is made at point A (left eye), according quantum theory this means that the complete state of the system has collapsed at all times for $\mathbf{B}$ ?

Figure 3. When an where exactly does the state function of the system collapse?
1. What we can say, in this case, about spacelike separation?

2. The measurement process is simultaneously (left eye and right eye, Fig. 3)?

3. Where the entanglement take place (Fig. 4)?

4. What we can say about the notion of spacetime?

We will argue in Section V, that "real" physical process of the quantum entanglement start from an implicate order (Bohm, 1980) towards the relevant explicate order. This is in the "inverted", "opposite", or "reverse" direction with respect to the classical quantum entanglement explanation, which from an explicate order to the related implicate order. Concisely, we argue that the entanglement takes place in the implicate order before the subject's visualization (subjective experience),i. e., the brain processes involved before conscious subjective experience. The subjective experience (SE) can be considered as an explicate order, which seems to involve disentanglement, and the physical neural network in the brain (the neural correlate of the related SE) seems to be responsible because of the decoherence via the interaction of the brain's cognitive frontoparietal feedback signals with environmental stimulus dependent feed forward signals (Vimal, 2010a). The concept of implicate/ explicate order can be applied at any level of complexity. In other words, in the above example we considered an implicate order at neural-network level (such as chitta or memory (Caponigro et al., 2010) formation and recall, attention, re-entry, brain processes involved in bringing the system to wakefulness and so on). One could argue that this implicate order is the explicate order for lower levels (such as neural-signal, neurotransmitter, synapticlevel, chitta/memory-formation processes, and genetic-level that are responsible for the formation of chitta/memory), which, in turn, has implicate order in terms of elementary particles. The fermions and bosons (elementary particles) are explicate orders and strings (string theory) or loops (loop quantum gravity) are related implcate orders. These can be considered as explicate orders and related sub-quantum field, unified field (consciousness unfied with all four fundamental forces), or Paramatman as 
fundamental Implicate Order (Vimal 2009a; 2009b; 2010a 2010b; 2010c; 2010d).

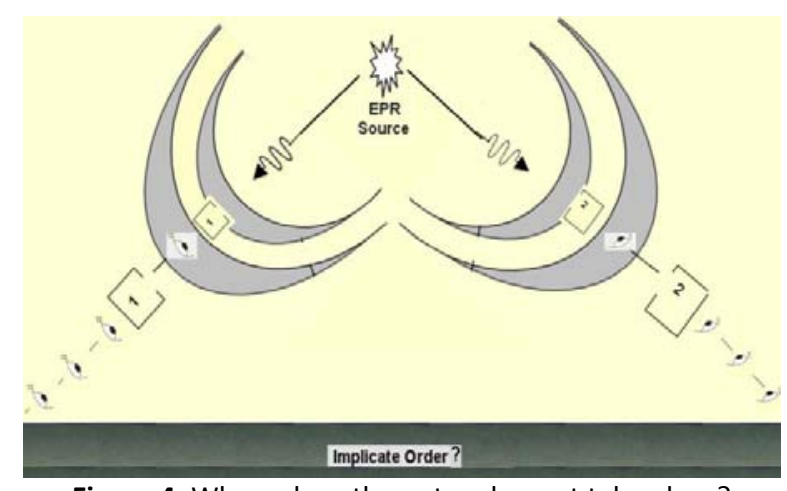

Figure 4. Where does the entanglement take place?

\section{Quantum Entanglement}

Entanglement is one of the most fundamental features in quantum mechanics. From an information theoretic point of view, the most remarkable feature of entanglement is the fact that in a maximally entangled state, all information is encoded in joint properties of the individual systems while the individuals themselves carry no information whatsoever (Zeilinger, 2000). From a phenomenological point of view, the phenomenon of entanglement is quite simple. When two physical systems come to an interaction, some correlation of a quantum nature is generated between the two of them, which persists even when the interaction is switched off and the two systems are spatially separated. Entanglement can be also created without direct interaction between the subsystems, via the so-called entanglement swapping (Zeilinger, 2000). Quantum entanglement describes a non-separable state of two or more quantum objects and has certain properties which contradict common physical sense. While the concept of entanglement between two quantum systems, which was introduced by $\mathrm{E}$. Schrödinger in 1935, is well understood, its generation and analysis still represent a substantial challenge. Especially entanglement between objects of different nature like atoms and photons was achieved only very recently (Zeilinger, 2000) and is subject of current research. The quantification of entanglement is a long standing problem in quantum information theory. Any good measure of entanglement should satisfy certain conditions. An important condition is that entanglement cannot increase by local operations and classical communications. Entanglement can be realized by having two entangled particles emerge from a common source, or by allowing two particles to interact with each other. Yet, another possibility to obtain entanglement is to make use of a projection of the state of two particles onto an entangled state. This projection measurement does not necessarily require a direct interaction between the two particles. When each of the two particles is entangled with another particle, an appropriate measurement (for example, a Bell-state measurement) of the partner particles will automatically collapse the state of the remaining two particles into an entangled state. This striking application of the projection postulate is referred to as entanglement swapping or teleportation of entanglement. Also, we can have, atomphoton entanglement. When a single atom is prepared in an excited state $|e\rangle$ it can spontaneously decay to the ground level $|g\rangle$ and emit a single photon. Due to conservation of angular momentum in spontaneous emission the polarization of the emitted photon is correlated with the final quantum state $|g\rangle$ of the atom. For a simple two-level atom, after spontaneous emission, the system is in a tensor product state of the atom and the photon. But for multiple decay channels to different ground states the resulting state of atom and photon is entangled. The physical process of spontaneous emission can not be explained by a semiclassical treatment of the light field but only by a quantum field approach. Today, a considerable effort is put into the research on entanglement with no restriction too, but a strong emphasis on, two-level systems, i.e. qubits. Bipartite entanglement is well understood and has been prepared in many different physical systems (Zeilinger, 2000). The math definition of entanglement varies depending on whether we consider only pure states or the general set of mixed states. Only for pure states, we say that a given state $|\psi\rangle$ of $n$ parties is entangled if it is not a tensor product of individual states for each one of the parties, that is,

$|\psi\rangle \neq\left|v_{1}\right\rangle_{1} \otimes\left|v_{2}\right\rangle_{2} \otimes \cdots \otimes\left|v_{n}\right\rangle_{n}$ 
For instance, in the case of 2 qubits $A$ and $B$ (sometimes called "Alice" and "Bob") the quantum state

$$
\left|\psi^{+}\right\rangle=\frac{1}{\sqrt{2}}\left[\left(|0\rangle_{A} \otimes|0\rangle_{B}+|1\rangle_{A} \otimes|1\rangle_{B}\right)\right]
$$

is entangled since $\left|\psi^{+}\right\rangle \neq\left|v_{A}\right\rangle_{A} \otimes\left|v_{B}\right\rangle_{B}$. On the contrary, the state

$$
\begin{aligned}
& |\phi\rangle=\frac{1}{2}\left[\left(|0\rangle_{A} \otimes|0\rangle_{B}+|1\rangle_{A} \otimes|0\rangle_{B}\right.\right. \\
& \left.\left.+|0\rangle_{A} \otimes|1\rangle_{B}+|1\rangle_{A} \otimes|1\rangle_{B}\right)\right]
\end{aligned}
$$

is not entangled, since

$$
|\phi\rangle=\left(\frac{1}{\sqrt{2}}\left(|0\rangle_{A}+|1\rangle_{A}\right)\right) \otimes\left(\frac{1}{\sqrt{2}}\left(|0\rangle_{B}+|1\rangle_{B}\right)\right) .
$$

A pure state like the one from Eq.2 is called a maximally entangled state of two qubits, or a Bell pair, whereas a pure state like the one from Eq. 4 is called separable.

In the general case of mixed states, we say that a given state $\rho$ of $n$ parties is entangled if it is not a probabilistic sum of tensor products of individual states for each one of the parties, that is,

$\rho \neq \sum_{k} p_{k} \rho_{1}^{k} \otimes \rho_{2}^{k} \otimes \cdots \otimes \rho_{n}^{k}$,

with $\left\{p_{k}\right\}$ being some probability distribution. Otherwise, the mixed state is called separable. The essence of the above definition of entanglement relies on the fact that entangled states of $n$ parties cannot be prepared by acting locally on each one of the parties, together with classical communication (telephone calls, e-mails, postcards...) among them. As said before, this set of operations is often referred to as "local operations and classical communication", or LOCC. Entanglement is a genuine quantum-mechanical feature which does not exist in the classical world. It carries non-local correlations between the different parties in such a way that they cannot be described classically.

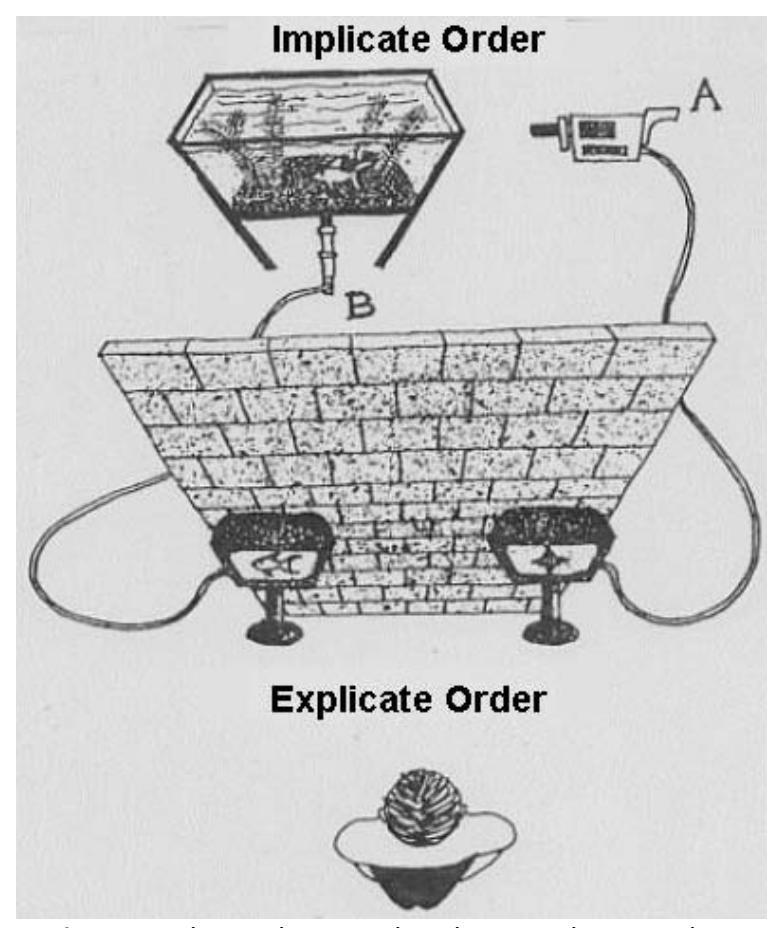

Figure 5. The Implicate and Explicate Order according David Bohm.

\section{The Implicate Order}

Today, most important studies in these fields are carried forward by Paavo Pylkkänen (Pylkkänen, 2007) and Basil Hiley (Hiley, 2000). Bohm, calls implicate order the primary reality, this reality exists 'folded up' in nature and gradually unfolds as the universe evolves, enabling organization to emerge, in this way, the implicate becomes explicate over time. Against the Copenhagen interpretation of quantum mechanics, he began to suspect that there was a deeper order underlying the complex behavior of particles, giving rise to his theory of an 'implicate order' in the universe, an 'undivided wholeness' that governs all reality. The theorization of the existence of a 'quantum potential', which determined the motion of particles was the main support for the existence of the implicate order. According Bohm (Bhom, 1980) the whole universe can be thought of as a holomovement (Fig.5), in which a total order is contained. The explicate order is a projection from higher dimensional levels of reality, and the apparent stability and solidity of the objects and entities composing it are generated and sustained by a ceaseless process of enfoldment and unfoldment, for subatomic particles are constantly dissolving into the implicate order and then 
recrystallizing. According to Globus (2007) implication the ordinary Cartesian order is Bohmian wholeness is dynamical: a enfolded to the whole where it "exists" in continuous holomovement. The potentia, while in explication the Cartesian holomovement has two simultaneous order is unfolded from the whole. processes: implication and explication. In

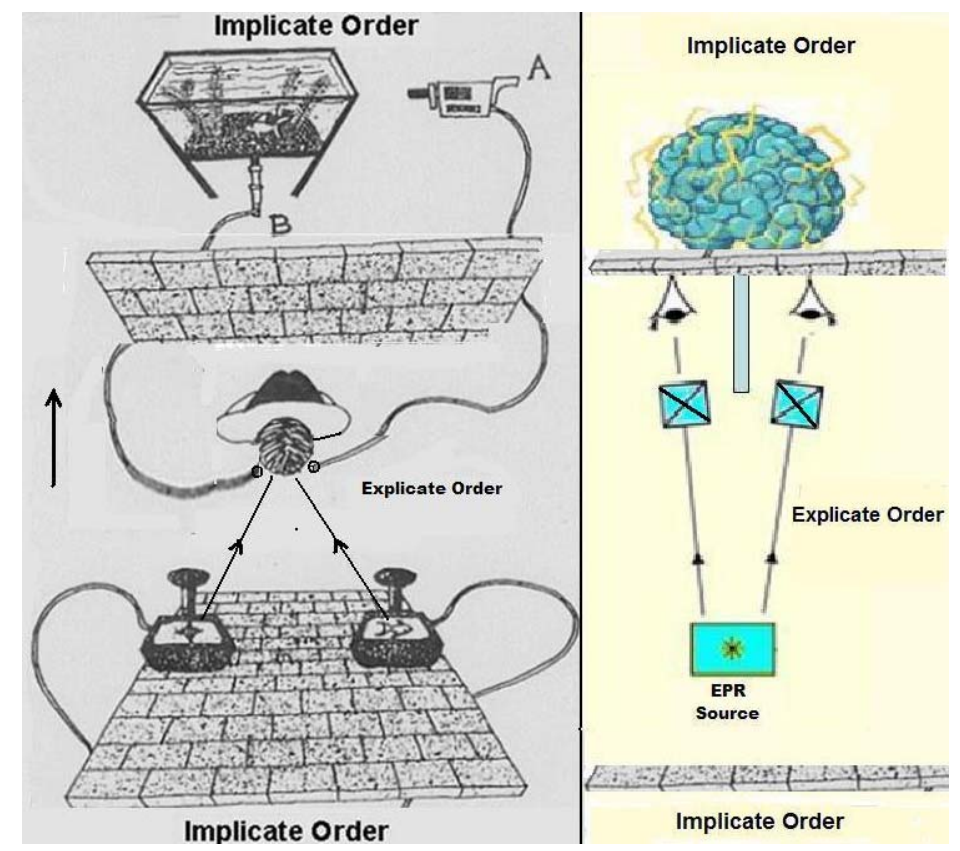

Figure 6. We are perennially "immersed" in the Implicate Order according our thesis.

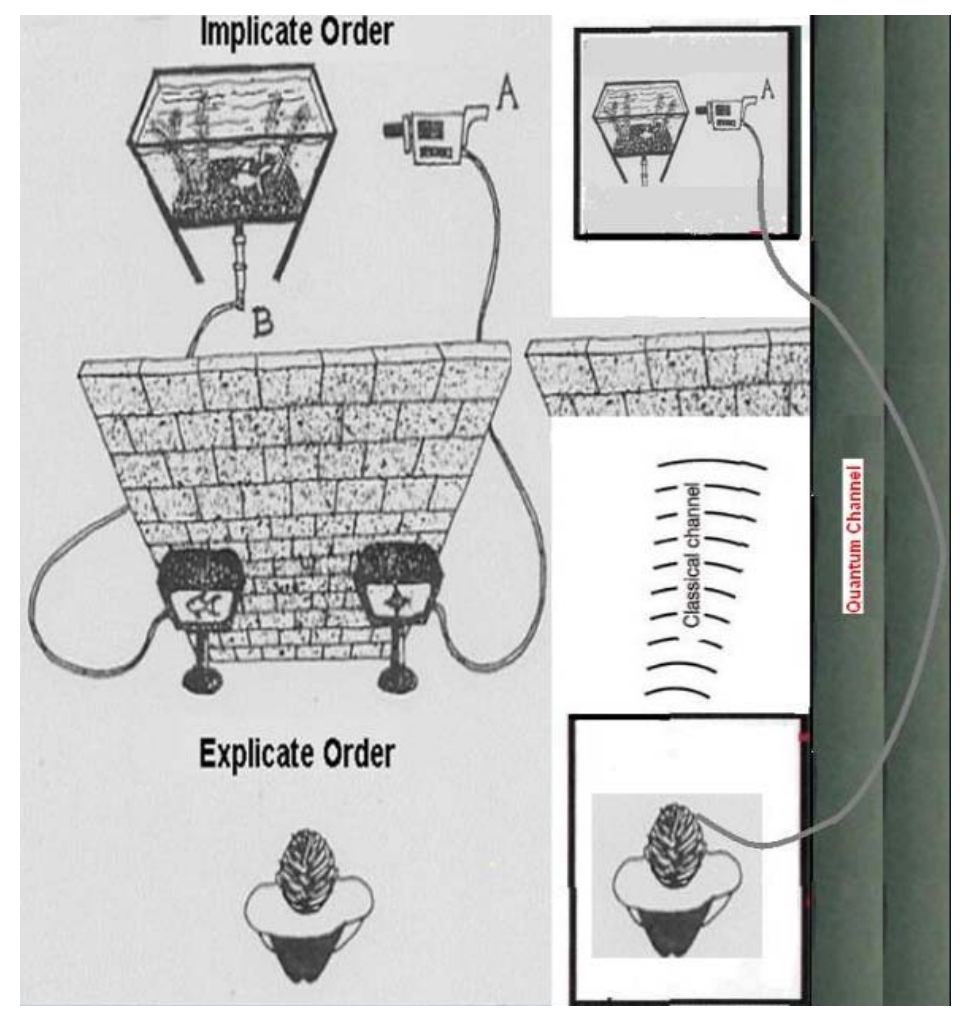

Figure 7. We named "quantum channel" the hidden implicate order. 


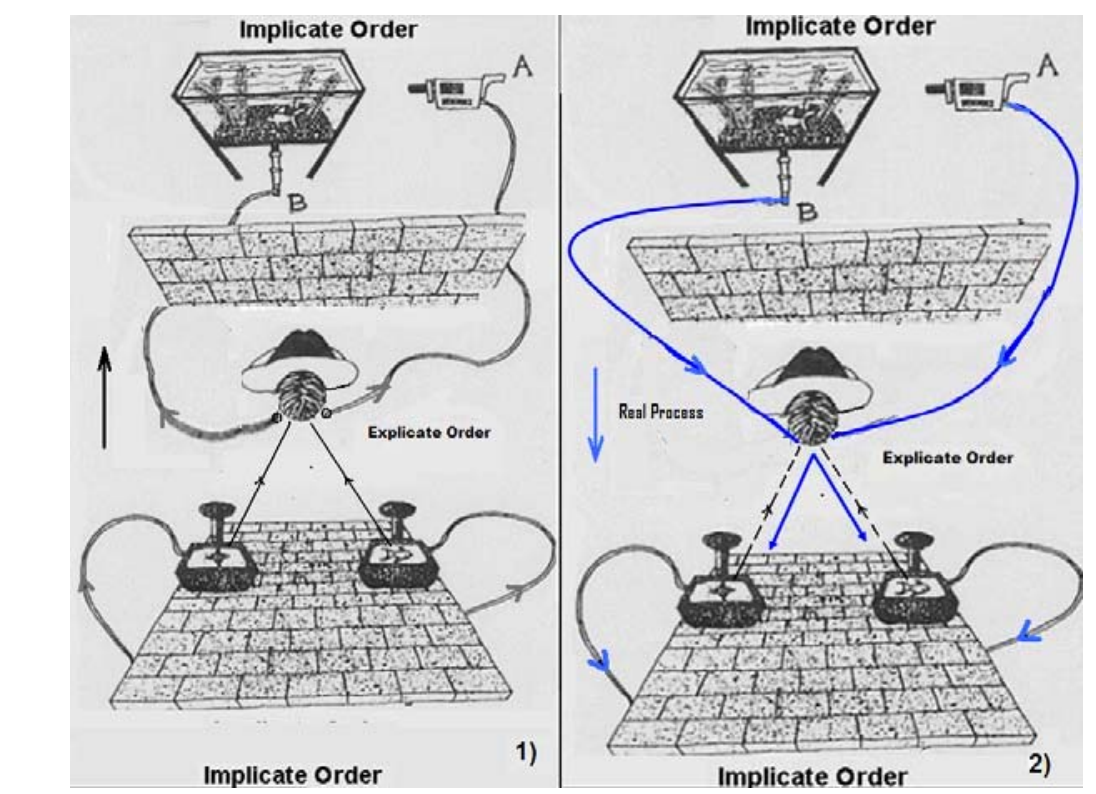

Figure 8. 1. Classic quantum entanglement, 2. Disentanglement the real physical process: from implicate order towards explicate order by mind.

\section{Philosophical Speculations: the "real" process of quantum entanglement}

In order to give a correct context of describing the underlying physical reality, according our analysis (Fig.6), it is necessary to review the notions of space-time, quantum entanglement phenomenon, implicate / explicate order and decoherence. We retain that physical reality (explicate order) must be linked to:

1. The foundation of implicate order has a "structure" without any spatial and temporal connotation.

2. The physical brain is responsible of disentanglment.

3. The phenomenon of decoherence as a means to produce classicity: the explicate order (Fig.7).

4. The "real" path of quantum entanglement is from implicate order towards explicate order. This is the "opposite" or "reverse" direction with respect to the classicalquantum concept of entanglement. In other words, the classical-quantum entanglement is from explicate order towards implicate order (Fig.8).

To sum up, we retain that the entanglement takes place before subject's visualization or subjective experience in the implicate order. The visualization (the explicate order) has to do with disentanglement and the brain is responsible for that. This view fits well with our previous paper (Caponigro et al., 2010) on the possible relationship between implicate order and Chitta or memory, a fundamental category in the Vedic theory of Mind.

\section{Implicate/ Explicate Order, Vedic Science, and the Dual-Aspect-Dual- Mode PE-SE Framework}

According to (Caponigro et al., 2010); the five Vedic entities Chitta, Manas, Buddhi, Ahamkara, and 'Paramatman $\rightarrow$ Atman $\rightarrow$ Purush $\leftrightarrow$ Prakriti $\rightarrow$ Brahma $\rightarrow$ Jivatman' or 'Paramatman $\rightarrow \quad$ ParamPurush / MahaPurush $\leftrightarrow$ Prakriti $\rightarrow$ ParamBrahma $\rightarrow$ Atman/Jivatman' are assimilable with Bohm's Implicate and Explicate order at various levels and the holomovement framework. For example, the entities 'Paramatman $\rightarrow$ Atman $\rightarrow$ Purush $\leftrightarrow$ Prakriti can be considered equivalent to Bohm's enfolded Implicate Order at fundamental level; whereas Brahma, Jivatman, Chitta, Manas, Buddhi, and Ahamkara can be considered as unfolded Explicate Order at various levels. For example, Chitta is assimilable with the holomovement that does not have the structure of space-time; the holomovement (via entanglement) unfolds and enfolds via space-time; in the same way Chitta unfolds and enfolds (via entanglement) with Manas, 
which represent the Explicate Order of Vedic theory of Mind. Bohm and J. Krishnamurthi (Vedic scholar and a great Indian thinker) communicated with each other, and Bohm's Implicate/ Explicate and enfolding/ unfolding is a sort of linked with the dual-aspect part of Vedic science; he is clearly a dual-aspect philosopher, which is consistent with the dual-aspect-dual-mode PE-SE framework (Vimal 2008; Vimal 2010a). Stapp's framework is closer to Dvait Advait Vedic framework (Stapp, 2005).

Vamadeva (David Frawley) suggested that we would have to discriminate between individual chitta or mind from cosmic chitta or cosmic mind, Mahat or cosmic buddhi and Chit or pure consciousness beyond manifestation. If quantum mechanics could find evidence of either chitta on a cosmic level or chit as the unmanifest background of the cosmos, it would be quite a breakthrough. To address Vamadeva's comment, we propose the following hypothesis: In dual-aspect-dual-mode PE-SE framework, individual chitta (memory bank), manas (different from western term "mind"), buddhi, ahankar are part of single brain/mind based individual consciousness, which is based on the interaction between environment (in terms of stimulus dependent feed forward signals along quantum and classical pathways) and individual cognition (attentional feedback signals) as detailed in (Vimal, 2009a). When many brains/minds and respective environments interact, social or group consciousness emerges (such laws, political views, religion, economics, and so on). When all brains/minds of all living beings (human, animals, plant life, and so on) and their respective environments interact, higher order cosmic consciousness (cosmic chitta, cosmic manas, Mahat or cosmic buddhi, and Mahat ahankar; or pure consciousness beyond manifestation or dual-aspectShivaShakti) emerges. One could argue for Trika Kashmir Shaivism where Shiva (mental aspect) and Shakti (physical aspect) are the two aspects of same entity. Dvait-Advait Vedanta is closer to Stapp's orthodox quantum mechanics framework (Stapp 1993; 1995; 1996; 1997; 1999; 2005; 2001; 2002; 2004a; 2004b; 2006; 2007; 2009a; 2009b; 2009c; 2009d; 2010a; 2010b). According to Stapp (2009b), "Von Neumann (orthodox) quantum mechanics is thus dualistic in the pragmatic and operational sense that it involves aspects of nature that are described in physical terms and also aspects of nature that are described in psychological terms, and these two parts interact in human brains in accordance with laws specified by the theory. This is all in close accord with classic Cartesian dualism. On the other hand, and in contrast to the application to classical mechanics, in which the physically described aspect is ontologically matterlike, not mindlike, in quantum mechanics the physically described part is mindlike! So both parts of the quantum Cartesian duality are fundamentally mindlike. Thus quantum mechanics conforms at the pragmatic/ operational level to the precepts of Cartesian duality, but reduces at a deep ontological level to a fundamentally mindlike nondual monism." Although, so far, there is no concrete quantum mechanics evidence of either chitta on a cosmic level or chit as the unmanifest background of the cosmos, one could argue that quantum bounce model of loop quantum gravity suggest that universe may have some memory (chitta) of previous big-bounce cycle and maintains recall, although it is controversial (Bojowald, 2008a; 2008b; Corichi et al., 2008a; 2008b; Vimal 2010b; 2010c; 2010d). In addition, if Stapp's framework is correct, then perhaps mind-brain dualism at pragmatic / operational level reduces at a deep ontological level to a fundamentally mindlike nondual monism at cosmic level. This is somewhat consistent with Trika Kashmir Shaivism with dual-aspect Shiva-Shakti framework at cosmic level (Vimal, 2009).

\section{Are Meditative Experiences a unique form of Quantum Entanglement?}

The scientific involvement in meditation in the past decade can be easily termed as a revolution. Meditation has received acclamation in the medical field as well as basic cognitive fields. However, the phenomenological aspects of meditation have been paid much less attention. Especially, meditative experiences are uniquely varied and mystically intriguing. Inner light perception is one of such experiences, which have its mention in wide varieties of Buddhist and Indian meditation 
techniques. Inner light perception has philosophical, neurological and quantum mechanical aspects, which makes it an especially unique meditative experience. We have addressed else where that this experience arises several important questions regarding the most basic domains like realism, information and observerproblem.

In this essay, we are especially interested in considering meditation as a unique form of quantum entanglement. As has been mentioned above, a quantum entanglement has following properties:

1. It occurs between two or more quantum entities,

2. It specifies an inseparable condition between the two quantum objects,

3. It occurs (probably) in the implicate order before the occurance of subjective experience.

With these concepts in mind, we now move towards the task of exploring the philosophical aspects of inner light perception and try to correlate it with the phenomena of quantum entanglement.

\subsection{Significance of Inner Light Perception in Meditation Philosophy} Although inner-light perception has been recorded in almost all of the religious practices, scientifically it has been studied in two meditation techniques:

1.Zen meditation technique (Huang, 2003; Chang and Lo, 2006), 2.Vihangam Yoga meditation technique (Prakash, 2009; Prakash et al., 2009).

In both of these meditation techniques, the experience of inner light perception is considered as a special one. For example, in the Zen meditation technique, the experience of inner light is considered as the highest stage of meditation. According to the Zen theory, bio-energy of a human being can be purified via Zen-meditation practice. This kind of energy transcends from the realqi,the spiritual-qi, the electrical-qi, and eventually to the light-qi. The highest level of qi, light-qi, can be experienced during the deep Zen-meditation.

This state, known in Zen-Buddhist philosophy as "Anuttara Samyak Sambodhi" translated, means that: There is nothing (an) more supremely high (uttara) than unities fullfillment (sam [samma']) of Being (yakkha, could also say Essence, or "soul" in loose sense) in the oneness (San. [samma, or Sva]) of exquisite perfection (sam, in combination with Bodhi is always a modifier of excellence) in great wisdom As per the Zen philosophy, the state of inner-light perception is the highest level of purified state of mind where it detaches itself from the sensory experiences and reaches the ultimate reality and the truest wisdom. This type of experience has been term as enlightenment, where the individual acquires knowledge of god (Bramhagyana) and loses the contact with sensory apparatus to come in equity with the supreme god (Teaching letter, 2008; Warner, 2000). Similarly in the Vihangam Yoga meditation, the attainment of inner-light perception, popularly known as Samadhi has been described as a state of equanimity of soul with god or universal consciousness which is all-pervading( Prakash, 2009; Prakash et al., 2009). The term Yoga infact literally means "addition" of the soul consciousness with the ultimate consciousness. This state, variously described as the "Hamsa awastha" or "Samadhi" is a state, where the individual loses his identity (mainly as a separation from his senses) and merges with the infinite bliss of the supreme power or God, which is all pervading in this universe. Thus inner light perception has been denoted as the highest level of meditation and considered very special.

\subsection{Inner Light Perception as Quantum Entanglement}

We have argued elsewhere that inner light perception is compatible with the philosophies of quantum mechanics, in the contexts of realism, information and reduction (Prakash et al., 2009). However, we did not specify the quantum phenomena to which it corresponds closely enough. Here we argue that the meditative experience of inner light perception is a unique movement of the quantum entity of consciousness from the explicit order of perception to the implicit order. Almost all the religious texts describe God as an all-pervading entity, which doesn't manifest itself directly but only in the form of its influences on the other manifest entities. The Vedic theory of god is 
very similar to the implicate order in the Bohm's construct in following ways:

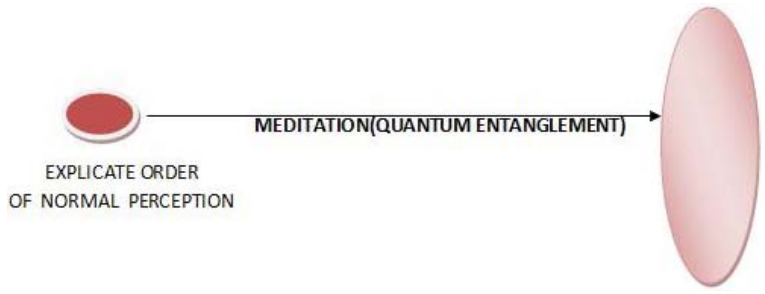

Figure 9. Implicate order of God-Energy.

1. God pervades all the objects and is the base of all the things

2. God never manifests itself but it affects those which are manifested (in the explicit order)

3. The God energy remains in undivided wholeness form

4. The creation of universe occurs by subtle movements in this God energy (Kampa).

Similarly, in Buddhism and ZenBuddhism, there are concepts of a similar entity, which is all pervading and which functions as a base for all other evident entities. This entity has been called as Buddha-form (Sarvam Budhtvam-Every thing is nothing but Buddha). Thus, the godenergy entity can be seen as an analogue of Bohm's implicate order (see Fig.9). Thus the process of meditation is then a movement from gross perception towards subtle levels of perception where the quantum entity of soul consciousness is able to "see" the implicate order of god-energy by meditation, which is then a quantum entanglement process from explicate to implicate order.

\section{Commentaries}

There could be different interpretations depending on the metaphysical frameworks. Our framework is the dual-aspect dual-mode PE-SE framework (Vimal, 2009; Vimal 2008) that has the least number of problems, which is close to Trika Kashmir Shaivism, where Shiva (mental aspect) and Shakti (physical aspect) are the two aspect of the same entity.

The present formalism of quantum theory is not adequate, even when the equations work well in connection with applications. Therefore, from our point of view any philosophical implication and interpretation, respectively, that is based on conventional quantum theory is problematic and has obviously nothing to do with that what we call "truth".

If the definition of "truth" is mindindependent reality (MIR) then MIR is always unknown as per Kant (Kant, 1787). In physics, we assume MIR = mind-dependent reality (MDR), which is debatable and in our view incorrect. Application of quantum physics to consciousness is not new. For example, According to Stapp (2009b);

"Von Neumann (orthodox) quantum mechanics is thus dualistic in the pragmatic and operational sense that it involves aspects of nature that are described in physical terms and also aspects of nature that are described in psychological terms, and these two parts interact in human brains in accordance with laws specified by the theory. This is all in close accord with classic Cartesian dualism. On the other hand, and in contrast to the application to classical mechanics, in which the physically described aspect is ontologically matterlike, not mindlike, in quantum mechanics the physically described part is mindlike! So both parts of the quantum Cartesian duality are fundamentally mindlike. Thus quantum mechanics conforms at the pragmatic/operational level to the precepts of Cartesian duality, but reduces at a deep ontological level to a fundamentally mindlike nondual monism."

Stapp bypasses some of the problems of substance dualism, but it still has problems (Vimal, 2010a). This framework seems is the same or close to Dvait (dualism)/Advait (mentalistic monism) Vedanta (Vimal, 2009c). Our approach follows the dual-aspect-dual-mode PE-SE framework that has the least number of problems, namely just one problem of the justifiable brute fact of dual-aspect. In addition, we have theoretical support that physics is invariant under PE-SE transformation, as discussed in (Vimal 2010b; 2010c; 2010d). 


\section{Acknowledgments}

RLPV was partly supported by VP-Research Foundation Trust and Vision Research Institute research Fund. Authors would like to thank anonymous reviewers for their critical comments, suggestions, and grammatical corrections. RLPV is also affiliated with Dristi Anusandhana

\section{References}

Bohm D. Wholeness and the Implicate Order. London, Routledge, Kegan Paul, 1980.

Bojowald M. Comment on Quantum bounce and cosmic recall. http://arxiv.org/abs/0811.2790. 2008

Bojowald M. Harmonic cosmology. How much can we know about a universe before the big bang? Proc Roy Soc A 2008; 464: 2135-2150.

Bouwmeester D, Ekert A and Zeilinger A. The Physics of Quantum Information. Springer, 2000.

Caponigro M and Vimal RLP. Quantum Interpretation of Vedic theory of Mind an epistemological path and objective reduction of thoughts. Journal of Consciousness Exploration Research 2010; 1(4): 402-410.

Chang KM and Lo PC. F-VEP and Alpha-Suppressed EEG physiological Evidence of inner-Light Perception During Zen Meditation. Biomed Eng Appl Basis Comm 2006; 2(18): 1-7.

Corichi A and Singh P. Quantum bounce and cosmic recall. Phys Rev Lett 2008; 100(16): 161-302.

Corichi A and Singh P. Reply to Comment on Quantum Bounce and Cosmic Recall. http:// arxiv.org/abs/ 0811.2983. 2008

Globus G. Mind, Matter and the Implicate Order by Paavo T.I. Pylkkänen. NeuroQuantology 2007; 5(4): 396-399.

Mikhailov MI and Mikhailova NS. Key to the vedas, Ed. Publishing office Nevsky prospect Sanct-Peterburg, 2005.

Hiley BJ. Non-commutative Geometry the Bohm Interpretation and the Mind-Matter Relationship. Proc. CASYS, Liege, Belgium, 2000.

Kant I. Critique of Pure Reason. Werner Pluhar. Trans. Indianapolis, 1987

Lo PC, Huang ML and Chang KM. EEG alpha blocking correlated with perception of inner light during zen meditation. Am J Chin Med 2003; 31(4): 629-42.

Prakah R, Haq Z, Prakash O, Sarkhel S and Kumar D. Inner Light Perception of Vihangam Yogis. A Qualitative Study. Journal of Consciousness Studies 2009; 16(2-3): 124-140.

Prakash R and Caponigo M. Inner light perception as a quantum phenomenon. NeuroQuantology 2009; 7(1): 188-197.

Prasad R. Towards God Realization. Adhyatmic Yantralaya, Garwar, Dist-Ballia, U.P India, 1994.

Pylkkänen P. Mind, Matter and the Implicate Order. Springer Verlag, 2007.

Sekatski P, Brunner N, Branciard C, Gisin N and Simon C. Towards Quantum Experiments with Human Eyes as Detectors Based on Cloning via Stimulated Emission, Phys Rev Lett 2009; 103(11): 601-605.
Sansthana, A-60 Umed Park, Sola Road, Ahmedabad-61, Gujrat, India; Dristi Anusandhana Sansthana, c/o NiceTech Computer Education Institute, Pendra, Bilaspur, C.G. 495119, India; and Dristi Anusandhana Sansthana, Sai Niwas, East of Hanuman Mandir, Betiahata, Gorakhpur, U.P. 273001 India.

Shimony A. Aspects of Nonlocality in Quantum Mechanics in Mechanics at the Crossroads, Springer, 2007.

Stapp HP. Mind, Matter, and Quantum Mechanics. Berlin Springer-Verlag, 1993.

Stapp HP. Why Classical Mechanics Cannot Naturally Accommodate Consciousness but Quantum Mechanics Can. Psyche 1995; 2(5). Accessed date; J une 29, 2010.

http:// psyche.cs.monash.edu.au/v2/ psyche-2-05stapp.html.

Stapp HP. The hard problem: a quantum approach. Journal of Consciousness Studies 1996; 3(3): 194210.

Stapp HP. Science of Consciousness and the Hard Problem. The J ournal of Mind and Behavior 1997; 8(2-3): 171-194.

Stapp HP. Attention, intention, and will in quantum physics. Journal of Consciousness Studies 1999; 6(8-9): 43-64.

Stapp HP. Quantum Theory and the Role of Mind in Nature. Foundations of Physics 2001;31:1465-1499.

Stapp HP. The Basis Problem in Many-Worlds Theories. Can J Phys 2001; 80: 1043-1052.

Stapp HP. Mind, Matter, and Quantum Mechanics. Second Edition, Springer-Verlag, 2004.

Stapp HP.Quantum Leaps in Philosophy of Mind Reply to Bourget's Critique. Journal of Consciousness Studies 2004; 11(129): 43-49.

Stapp HP. Quantum Interactive Dualism: An Alternative to Materialism. Journal of Consciousness Studies 2005; 12(11): 43-58.

Stapp HP. Quantum Approaches to Consciousness. M. Moskovitch P. Zelazo Editions. Cambridge Handbook of Consciousness. Cambridge Univ Press, 2006.

Stapp HP. Mindful Universe: Quantum Mechanics and the Participating Observer. Berlin, Heidelberg, New York, US.: Springer, 2007.

Stapp HP. A Model of the Quantum-Classical and Mind-Brain Connections and the Role of the Quantum Zeno Effect in the Physical Implementation of Conscious Intent. Mind, Matter, and Quantum Mechanics. Springer, 2009.

Stapp HP. Nondual quantum duality. Accessed date, June 29, 2010. http:// www-physics.lbl.gov/ stapp/ stappfiles.html.

Stapp HP. Philosophy of Mind and the Problem of Free Will in the Light of Quantum Mechanics. In A. von Mueller (Ed.), On Thinking. Berlin, Heidelberg, New York: Springer, 2009.

Stapp HP. Quantum reality and mind. Journal of Cosmology, Published Online 2009. http://journalofcosmology.com/ QuantumConsciou sness105.html. Accessed date: J uly 29, 2010.

Stapp HP. Compatibility of Contemporary Physical Theory with Personality Survival. Accessed date 
July 29, 2010. http://www-physics.lbl.gov/ stapp/ Compatibility.pdf.

Stapp HP. The Effect of Mind upon Brain. In S. Koutroufinis-Life and Process. Towards a Whiteheadian Biophilosophy. Ontos-Verlag, 2010.

Torre A. Entanglement for all quantum states. Eur J Phys 2010; 31: 325-332.

Vimal RLP. Matching and selection of a specific subjective experience, conjugate matching and subjective experience. A Journal of Integrative Neuroscience 2008; 7(1): 49-73

Vimal RLP. Subjective Experience Aspect of Consciousness Part I - Integration of Classical, Quantum, and Subquantum Concepts. NeuroQuantology 2009; 7: 390-410.

Vimal RLP. Subjective Experience Aspect of Consciousness Part II. Integration of Classical and Quantum Concepts for Emergence Hypothesis. NeuroQuantology 2009; 7: 411-434.

Vimal RLP. Towards a Theory of Everything Part I Introduction of Consciousness in Electromagnetic Theory, Special and General Theory of Relativity. NeuroQuantology 2010; 8: 206-230.

Vimal RLP. Towards a Theory of Everything Part II Introduction of Consciousness in Schrödinger equation and Standard Model using Quantum Physics. NeuroQuantology 2010; 8(3): 206-230.

Vimal RLP. Pre-existence of Subjective Experiences in Type-B Materialism. Bridging Materialism and Anti-materialism via Dual-Aspect Optimal Framework. Vision Research Institute. Living Vision and Consciousness Research 2009; 2(2): 185

Vimal RLP. Proto-experiences and Subjective Experiences: Classical and Quantum Concepts. J ournal of Integrative Neuroscience 2008; 7: 4973.

Vimal RLP. On the Quest of Defining Consciousness. Forthcoming in Mind and Matter 2010. http:// sites.google.com/ site/ rlpvimal/Home/ 2010Vimal-DefineC-LVCR-3-2.pdf].

Vitiello G. Dissipation and memory capacity in the quantum brain model. International Journal of Modern Physics 1995; B9: 973-989.

Warner B. Hardcore Zen. Wisdom Publications, 2003. 